Leuk Lymphoma. 2016 November ; 57(11): 2489-2498. doi:10.1080/10428194.2016.1195500.

\title{
MUC1 in hematological malignancies
}

\author{
Dina Stroopinsky ${ }^{\mathrm{a}}$, Donald Kufe ${ }^{\mathrm{b}}$, and David Avigan ${ }^{\mathrm{a}}$ \\ aBeth Israel Deaconess Medical Center, Harvard Medical School, Boston, MA, USA \\ bDana Farber Cancer Institute, Harvard Medical School, Boston, MA, USA
}

\begin{abstract}
Mucin1 (MUC1) is a transmembrane oncogenic protein that plays a central role in malignant transformation and disease evolution, including cell proliferation, survival, self-renewal, and metastatic invasion. MUC1 has been shown to interact with diverse effectors such as $\beta$-catenin, receptor tyrosine kinases, and c-Abl, which are of importance in the pathogenesis of various hematological malignancies. In myeloid leukemia, MUC1 has been shown to have an essential role in leukemia stem-cell function, the induction of reactive oxygen species (ROS), and the promotion of terminal myeloid differentiation. As such, MUC1 is an attractive therapeutic target in hematologic malignancies. Targeting MUC1 has been shown to be an effective approach for inducing cell death in tumor in in vivo and in vitro models. Furthermore, MUC1 inhibition is synergistic with other therapeutic agents in the treatment of hematologic disorders. This review will explore the role of MUC1 in hematological malignancies, and strategies for targeting this oncoprotein.
\end{abstract}

\section{Keywords}

AML; MUC1; multiple myeloma; ROS

\section{Introduction}

Mucin1 (MUC1) is a heterodimeric-epithelial cell glycoprotein that is aberrantly expressed in diverse carcinomas and mediates multiple pathways critical for oncogenesis.[1-9]. Intriguingly for an epithelial cell-associated protein, MUC1 is also aberrantly expressed in hematologic malignancies.[10-19]. MUC1 is translated as a single polypeptide that undergoes autocleavage into two subunits, which in turn form a stable noncovalent heterodimer at the cell surface. The MUC1 N-terminal subunit (MUC1-N) contains glycosylated tandem repeats, which are characteristic features of the mucin family.[8,20]. MUC1-N forms a complex with the transmembrane MUC1 C-terminal subunit (MUC1-C). [20]. MUC1-C contains a 58-amino acid (aa) extracellular domain, a 28-aa transmembrane domain, and a 72-aa cytoplasmic tail. MUC1-C interacts with receptor tyrosine kinases, such

CONTACT Dina Stroopinsky, dstroopi@bidmc.harvard.edu, Beth Israel Deaconess Medical Center, Medicine, 330 Brookline Ave, Boston, 02215-5491, MA, USA.

Potential conflict of interest: Disclosure forms provided by the authors are available with the full text of this article at http:// dx.doi.org/10.1080/10428194.2016.1195500. 
as FLT3, at the cell membrane.[21]. MUC1-C also forms homodimers that are imported into the nucleus, where MUC1-C interacts with transcription factors, such as NF-kB p65 and the $\beta$-catenin/TCF4 complex.[22-24]. MUC1-C is also transported to the mitochondrial outer membrane, where it functions in blocking the intrinsic apoptotic pathway.[25-27]. In these ways, MUC1-C has been shown to mediate critical aspects of oncogenesis, including cell proliferation, autonomous self-renewal[28], tissue invasion[22,29], and resistance to apoptosis and cytotoxic injury.[25,30,31]. Additionally, our laboratory has recently identified that MUC1 may play an important role in modulating the immunosuppressive milieu of the tumor microenvironment.[32]. The role and function of MUC1 in hematologic malignancies are discussed below and we highlight its potential as a novel therapeutic target for blood cancers.

\section{MUC1 expression in hematological malignancies}

\section{Lymphoma}

Our group has demonstrated that MUC1 is strongly expressed in Sezary and Mycosis Fungoides cells lines, as well as patient derived primary cutaneous T cell lymphoma (CTCL) samples.[10]. In contrast, T cells isolated from healthy volunteers lack MUC1 expression, suggesting that MUC1 may play a pivotal role in the evolution of T cell lymphoma.[10]. Consistent with these findings, we demonstrated that in patients with circulating $\mathrm{T}$ cell lymphoma cells, MUC1 expression is restricted to the malignant clonal population as identified by a unique TCR Vb chain signature. In contrast, MUC1 expression was absent in the polyclonal normal $\mathrm{T}$ cell population. Dyomin et al. described a case of extra nodular B cell lymphoma with a $\mathrm{t}(1 ; 14)(\mathrm{q} 21 ; \mathrm{q} 32)$ translocation and overexpression of MUC1 mRNA and protein.[33]. However, we and others have found little if any MUC1 expression in B cell lymphomas, including cell lines derived from Large Cell Lymphoma, Burkitt's Lymphoma, and Mantle Cell Lymphoma.[10].

\section{Leukemia}

MUC1 is overexpressed in human acute myelogenous leukemia (AML) cell lines and a majority of primary samples obtained from AML patients at time of presentation and relapse.[16,19,34]. MUC1 is expressed at higher levels in CD34+ cells derived from AML patients as compared to those in cord blood samples.[19]. MUC1 has also been detected in the CML myeloid blast crisis cell line (K562) and in primary samples from patients with blastic transformation, in contrast to chronic phase disease.[18,35,36].

AML arises from a malignant stem cell population characterized by dormancy, intrinsic resistance to cytotoxic injury, and the capacity for self-renewal. Leukemia stem cells represent a critical reservoir for disease relapse. Importantly in this regard, our laboratory has demonstrated that MUC1 is differentially expressed by AML stem cells as compared to normal hematopoietic stem cells. Analysis of bone marrow samples derived from 20 patients with active AML showed consistent expression of MUC1 in the stem cell (CD34+/lineage-/ CD38-) and early progenitor (CD34+/lineage-/CD38+) subpopulations. In contrast, $\mathrm{CD} 34+/$ lineage-/CD38 - cells from healthy donors express MUC1 at low to undetectable levels.[16]. 


\section{Multiple myeloma}

Overexpression of MUC1 has been observed in multiple myeloma (MM) cell lines and primary patient-derived samples, and is associated with a poor prognosis.[12,14,17].

Aberrant glycoforms of MUC1 are found in the majority of both immature (CD38++/ $\mathrm{CD} 45+$ ) and mature (CD38++/CD45-) plasma cells obtained from bone marrow of patients with $\mathrm{MM}$, but are not observed in bone marrow-derived plasma cells obtained from normal donors.[12].

\section{Functional role of the MUC1 oncoprotein in hematological malignancies MUC1-C inhibition and induction of reactive oxygen species (ROS)}

MUC1 suppresses reactive oxygen species (ROS) that normally accumulate in cells undergoing stress or injury.[37]. This function is vital in order to maintain the malignant phenotype in which oxidative stress is induced due to rapid cell proliferation, hypoxia and cytotoxic injury. This function is mediated by the MUC1-C cytoplasmic domain and, at least in part, the induction of genes which confer protection against the disruption of redox balance.[17,18,36,38].

To further interrogate this mechanism in the setting of hematological malignancies, our group has employed the use of a small molecule inhibitor of MUC1-C signaling. Localization of MUC1-C to the nucleus is dependent on homodimer formation through a CQC motif in the MUC1-C cytoplasmic tail [Figure 1]. Accordingly, the cell-penetrating peptide GO-203 was developed to bind the CQC motif, thereby blocking MUC1-C homodimerization and function.[39].

In multiple hematological malignancy models including CML, AML, MM, and CTCL, targeting MUC1-C with GO-203 is associated with a marked increase in hydrogen peroxide and superoxide.[10,34,38]. In addition, GSH levels are downregulated in the response to MUC1-C inhibition, as a result of the induction of oxidative stress and downregulation of TIGAR. Accordingly, restoration of GSH levels with NAC, a precursor of GSH synthesis, attenuated the increases in ROS induced by the MUC1-C inhibitor.[10,18].

Previous studies have demonstrated that the self-renewal of normal hematopoietic cells is critically dependent on ROS.[40]. In this context, the self-renewal of AML cells appears to be sensitive to enhanced ROS levels, suggesting that the disruption of redox balance in AML cells has potential as a therapeutic strategy.[34]. Indeed, inhibition of MUC1-C and the associated increases in ROS induce late apoptosis/necrosis of AML cell lines and patient derived tumor cells. Significantly, the initiation of AML cell death by MUC1-C inhibition is reversed in large part by NAC, confirming the involvement of increases in ROS levels in cell death. Similar results were observed in a CML model.[18,34].

In MM and CTCL models, MUC1-C inhibition by in vitro exposure to GO-203 is also associated with an increase in ROS levels. MUC1 inhibition was also associated with the down-regulation of the TP53-induced glycolysis and apoptosis regulator (TIGAR). [10,38,41]. Like MUC1-C, TIGAR decreases intracellular ROS levels and protects against ROS-induced cell death. TIGAR inhibits glycolysis and stimulates the pentose phosphate 
pathway, effectively lowering fructose-2,6-bisphosphate levels in cells and decreasing intracellular ROS levels, at least in part, through the conversion of NADP to NADPH. Inhibition of MUC1-C decreases TIGAR protein without a detectable effect on TIGAR mRNA levels; MUC1-C could potentially contribute to stability of the TIGAR protein, such that inhibition of MUC1-C promotes TIGAR turnover. Alternatively, MUC1-C regulates the expression of miRNAs, one or more of which could block TIGAR translation. Furthermore, treatment of MM and CTCL cells with the MUC1-C inhibitor GO-203 in the presence of the antioxidant NAC abrogated the suppression of TIGAR, indicating that this response is also mediated by oxidative stress as a positive feedback loop in which MUC1-C and TIGAR function in concert to regulate redox balance.[10,38,42].

\section{Role of MUC1 in mediating AML stem cell function}

MUC1 is selectively expressed by leukemia stem cells as compared to normal hematopoietic stem cells.[16,19]. To assess the functional consequence of this observation, we assessed whether MUC1 expression is associated with efficient engraftment of primary human AML cells in a xenograft murine NOG model. CD34+/lineage-MUC1 high expressing cells were segregated from CD34+/lineage-/MUC1 low cells by flow cytometric sorting. Of note, both populations exhibited the cytogenetic abnormality representative of malignant clone, but the MUC1 low population also contained a small subpopulation of normal stem cells. Mice inoculated with $\mathrm{CD} 34+/$ lineage-/MUC ${ }^{\text {high }}$ expressing cells demonstrated rapid and highly efficient AML engraftment in the absence of normal hematopoietic elements. In contrast, challenge of animals with the CD34+/lineage-/MUC1 ${ }^{\text {low }}$ cells did not develop AML, but showed evidence of mixed myeloid and lymphoid normal hematopoietic engraftment.[16]. These findings indicate that MUC1 is tightly correlated with the potential for leukemic engraftment by progenitor populations, supporting a functional role in maintaining the malignant stem cell phenotype.

\section{MUC1 mediates differentiation arrest in myeloid malignancies}

Disruption of normal patterns of differentiation is a critical aspect in the development of AML. The potential role of MUC1 in mediating maturation arrest is supported by the observation that MUC1 expression is markedly upregulated with transformation of CML to blast crisis, a process associated with the rapid accumulation of primitive progenitors and loss of the capacity for undergoing differentiation, which is a characteristic of chronic phase disease.[35]. Consistent with these findings, the silencing of MUC1 in CML cell lines is associated with decreased capacity for self-renewal and differentiation towards an erythroid phenotype.[35]. Moreover, inhibition of MUC1-C with GO-203 results in the arrest of CML cell growth, induction of myeloid differentiation, and loss of survival. Similarly, we have demonstrated that MUC1 silencing in AML cells is associated with morphologic changes, cytokine production, and loss of engraftment capacity in murine models, findings consistent with induction of terminal differentiation. $[18,35,36]$.

The available evidence supports a model in which MUC1 blocks terminal myeloid cell differentiation by suppressing ROS and promoting proliferation and survival. Treatment of CML cells with agents that decrease self-renewal, such as 1-b-arabinofuranosylcytosine (ara-C) increases ROS and results in the irreversible induction of hemoglobin synthesis.[43]. 
ROS has also been shown to play a role in inducing myeloid differentiation of normal hematopoietic cells and leukemic blasts. In this way, CDDO, an agent that increases intracellular ROS, potently induces maturation of leukemic blasts.[44]. The inhibition of MUC1-C in myeloid leukemia cell lines and primary tumor cells has resulted in both maturation along the myeloid lineage and loss of self-renewal, consistent with induction of terminal differentiation dependent on the associated increase in ROS.

\section{MUC1 interaction with Wnt/ $\beta$-catenin}

The Wnt/ $\beta$-catenin pathway exerts diverse effects in support of the malignant phenotype, including induction of proliferation and resistance to apoptotic injury. MUC1-C binds directly to $\beta$-catenin [Figure 2], resulting in its stabilization and thereby aberrant activation of WNT target genes, such as $C C N D 1$ and $M Y C$, in carcinoma cells.[45,46]. In hematological malignancies, the interaction of MUC1 and $\beta$-catenin was demonstrated in AML and CML.[18]. The Wnt/ $\beta$-catenin pathway has been shown to be critical and associated with self-renewal and proliferative properties of leukemic, as compared to normal, hematopoietic progenitors.[23,47,48]. In addition, recent work has shown that MUC1-C drives MYC in MM cells by stabilizing b-catenin and activating the WNT pathway.[49].

\section{MUC1 FLT3 interaction}

FLT3 functions as an important oncogene in AML.[50]. FLT3 overexpression is noted in approximately one third of AML patients due to the presence of internal tandem duplications, which are associated with poor outcomes following standard chemotherapy. Liu et al. demonstrated that MUC1-C associates with mutant FLT3 in AML cell lines and primary AML cells. In this way, MUC1-C contributes to FLT3 activation in AML cells and targeting MUC1-C inhibits the mutant FLT3 signaling pathway. Additionally, targeting MUC1-C with GO-203 increases sensitivity of mutant FLT3 AML cells to treatment with FLT3 inhibitors.[21].

Interestingly, MUC1-C was shown to form complexes with both the WT FLT3 allele and the mutant FLT3-ITD allele. The MUC1-C subunit interacts with RTKs at the cell membrane resulting in phosphorylation of FLT3L and subsequent activation of downstream effectors. In contrast, targeting MUC1-C disrupts MUC1-C/FLT3 complexes, downregulates FLT3 activation, and suppresses downstream AKT, ERK, and STAT5 signaling. Of note, silencing MUC1-C was shown to be effective in inhibiting growth and inducing death of AML cells resistant to FLT3 inhibitors.[21].

\section{MUC1 Bcr-Abl interaction}

In a CML model, it was demonstrated that MUC1-C associates with the Bcr-Abl protein by binding directly to the Bcr N-terminal region of Bcr-Abl, thereby stabilizing the protein. [35]. Furthermore, MUC1-C was shown to directly bind and sequester c-Abl in the cytoplasm; the phosphorylation of MUC1-C on Tyr60 functions as a binding motif for the cAbl SH2 domain [Figure 2].[51]. 
MUC1 silencing in CML cells was associated with the down-regulation of Bcr-Abl expression. MUC1 had no effect on Bcr-Abl mRNA levels but MUC1 downregulation led to decreased stability of the Bcr-Abl protein. Furthermore, the stability of the endogenous Bcr p160 and p130 proteins was reduced due to MUC1 silencing, indicating that the effects of MUC1-C on Bcr-Abl stability may be mediated through the binding of MUC1-C to the Bcr $\mathrm{N}$-terminal region. On the other hand, MUC1-C has no apparent effect on the stability of the $\mathrm{c}-\mathrm{Abl}$ protein, but blocks nuclear targeting of $\mathrm{c}-\mathrm{Abl}$ in the apoptotic response to DNA damage.[51].

\section{Other signaling pathways}

MUC1-C interacts with various signaling molecules that include kinases - c-Src, Lyn, GSK3 $\beta$, PKC 8 [35, 51-53]; growth factor receptors - EGFR, ErbB2-4, FGFR3,[54-56] and transcription factors, such as ERa and p53 [Figure 2].[20,57,58].

Furthermore, recent data indicates that MUC1-C functions as a chaperone to hold proteins in certain configurations that regulate stability or activity. MUC1-C was shown to destabilize p53 and increase stability and activity of $\beta$-catenin and ERa.[57,58]. The MUC1-C cytoplasmic domain is phosphorylated by diverse kinases and binds directly to multiple effectors that have been linked to oncogenesis [Figure 2].[(9,20]. In this regard, the MUC1$\mathrm{C}$ cytoplasmic domain is an intrinsically disordered protein, as found in other oncoproteins, that has the plasticity to function as a node for the integration of multiple signaling pathways.[4].

\section{MUC1 as a therapeutic target in hematologic malignancies}

The MUC1-C cytoplasmic domain contains a CQC motif that is necessary for its homodimerization and subsequent nuclear localization. Based on these findings, our group has developed a cell-penetrating peptide drug to inhibit MUC1-C homodimerization and its oncogenic function. The MUC1-C inhibitor GO-203 contains the endogenous MUC1-C CQCRRKN amino acid sequence linked to nine arginine residues at the $\mathrm{N}$-terminus for cell permeability.[39]. MUC1-C inhibitors blocked the interaction between MUC1-C and its downstream targets in MM, lymphoma, and leukemia cells, leading to the induction of apoptosis and necrosis in vitro.[10,34,38]. In an immunocompromised murine model, administration of GO-203 was effective in eliminating established human U266 MM tumor xenografts.[17]. Similarly, treatment of NSG mice with GO-203 protected animals from an otherwise lethal challenge of primary AML cells.[16]. Most significantly, treatment of animals with established disease resulted in eradication of disease.[10,16]. Similarly, exposure of human CTCL cell lines and primary cells to GO-203 results in dose-dependent cell death. In a xenogeneic murine model, treatment with GO-203 significantly reduced to tumor bulk and improved survival in animals challenged with the human CTCL cell line. [10]. Interestingly, treatment of normal B cells and normal hematopoietic stem cells with MUC1-C inhibitor is not associated with loss of survival.[21]. Based on these findings, a phase I clinical trial of GO-203 in patients with relapsed or refractory AML is currently underway. 


\section{MUC1-C inhibition is synergistic in combination with standard chemotherapy}

\section{MUC1-C inhibition increases susceptibility of CML cells to imatinib}

Inhibition of Bcr-Abl with imatinib induces apoptosis of Bcr-Abl positive CML cells and is highly effective in treating patients with CML.[59]. However, the persistence of Bcr-Abl positive tumor cells in CML patients treated with imatinib has indicated that factors other than inhibition of the Abl kinase function may contribute to the pathogenesis of the disease. [60,61]. Kawano et al. have demonstrated that silencing MUC1 in CML cell lines increases sensitivity to imatinib-induced apoptosis.[35]. MUC1-C is targeted to the mitochondrial outer membrane by an HSP90 dependent mechanism and inhibiting the intrinsic apoptotic pathway.[25]. Destabilization of Bcr-Abl with HSP90 inhibitors was shown to sensitize CML cells to the induction of apoptosis through the intrinsic mitochondrial pathway in part due to its effects on trafficking of MUC1-C to the mitochondrial membrane. Moreover, based on studies in other tumor models, the increased sensitivity to imatinib could potentially be due in part to the MUC1-C induced stabilization of the mitochondrial permeability transition and a block in the release of apoptosis-promoting factors.[25].

\section{MUC1-C inhibition increases susceptibility of AML cells to FLT3 inhibitors}

Treatment of AML cells resistant to FLT3 inhibitors with GO-203 was associated with cell death, suggesting that targeting MUC1-C could be effective in the treatment of AML that is unresponsive to PKC412 or other FLT3 inhibitors. Moreover, GO-203 increases sensitivity of mutant FLT3 AML cells to FLT3 inhibitor treatment. Treatment with PKC412 alone has induced partial and transient responses in early clinical trials; therefore, GO-203 could be used in combination with PKC412 for the treatment of both FLT3 inhibitor-sensitive or inhibitor-resistant AML.[21].

\section{MUC1-C inhibition is synergistic with bortezomib in MM}

Bortezomib is a proteasome inhibitor that exhibits potent anti-myeloma activity and is approved as therapy for frontline and relapsed disease.[62]. However, disease resistance emerges with ongoing therapy.[62]. Previous studies have demonstrated that agents that increase oxidative stress (e.g. IGF-1R inhibitor) are synergistic with bortezomib in treatment of bortezomib resistant MM cells and render the cells more susceptible to bortezomib treatment.[63]. However, bortezomib resistance is associated with loss of ROS increase in response exposure to the proteasome inhibitor.

Yin et al. have demonstrated that bortezomib resistant MM cells remained sensitive to MUC1-C targeting.[42]. Both bortezomib resistant and drug-naïve MM cells responded to GO-203 with increases in ROS levels and induction of apoptosis and necrosis.[42]. Interestingly, treatment of MM cells with GO-203 in combination with bortezomib was associated with synergistic increases in ROS that were greater than the levels achieved by either agent alone.[42]. These results suggest that GO-203 re-sensitizes bortezomib resistant cells to bortezomib, which leads to increases in hydrogen peroxide and superoxides. Consistent with these findings, GO-203 treatment reversed resistance to bortezomib and the 
combination of GO-203 and bortezomib was synergistic in inhibiting growth and inducing cell death in bortezomib resistant MM cells. It was further demonstrated that exposure to NAC disrupts this effect, indicating that it is mediated by disrupting redox balance.[42].

\section{MUC1-C inhibition is synergistic with AraC in AML}

Our group has demonstrated that the MUC1-C cytoplasmic domain associates with the $\beta$ catenin/T-cell factor 4 (TCF4) complex[23,45], which regulates cell proliferation, differentiation, and apoptosis.[23]. Accumulation of $\beta$-catenin in the cytoplasm favors its translocation to the nucleus as a cofactor for TCF family transcription factors, thereby activating the transcription of $\mathrm{Wnt} / \beta$-catenin target genes. The MUC1-C oncoprotein facilitates nuclear translocation of active $\beta$-catenin to the nucleus, an event which is necessary for downstream signaling pathways. Silencing MUC1-C in AML cells and the resultant decline of $\beta$-catenin translocation to the nucleus results in a significant downregulation of survivin - a member of the inhibitor of apoptosis protein (IAP) gene family that inhibits apoptosis, enhances proliferation, and promotes angiogenesis expression.[64]. The downregulation of survivin expression has been shown to contribute to the reversal of drug resistance in acute leukemia models. MUC1-C inhibition renders AML cells more susceptible to cytotoxic injury from exposure to Ara-C. Concurrent treatment of AML cells with MUC1-C inhibitor GO-203 and Ara-C was found to have a synergistic effect.[64].

\section{MUC1 as a target for immunotherapy}

The aberrant and increased expression of MUC1 by multiple carcinomas and hematopoietic malignancies has established the two MUC1 subunits (MUC1-C and MUC1-N) as potential targets for the development of anti-cancer vaccines, antibodies, and small molecules.[15,6568]. Phase I and Phase II clinical trials are currently underway with recombinant vaccinia and fowlpox-based vaccines that express both MUC1 subunits.[8]. Additionally, liposomebased vaccines which incorporate the MUC1-N subunit are under clinical investigation for the treatment of hormone-sensitive breast cancer and non-small cell lung cancer.[69].

In MM, a phase I/II study is conducted using ImMucin, a 21-mer cancer vaccine encoding the signal peptide domain of MUC1. Vaccination was well tolerated and was shown to induce a robust diversified T- and B-cell ImMucin specific immunity in MM patients, resulting in least disease stabilization in most patients.[70].

Potential therapeutic agents have also been identified in monoclonal antibodies generated against the MUC1-N tandem repeats. However, the surface of the malignant cell sheds MUC1-N, allowing it to circulate in the plasma of patients with cancer; as a result, antiMUC1-N antibodies are therefore unable to reach their targets. Efforts to generate antibodies targeting the MUC1-C subunit are being pursued.

Previous work has demonstrated that neonatal $\mathrm{T}$ cells can be activated by varying the dose of antigens, adjuvants and the type of antigen presenting cells. Gong et al. have shown that using adult MUC1.Tg mice that are tolerant to MUC1 antigen, immunization with dendritic cell (DC)/tumor cell fusions expressing MUC1 was highly effective in inducing cellular and humoral immunity against MUC1.[71]. Immunization with the DC-based vaccine was 
shown to activate MUC1 reactive clones that are functionally inactive and thereby reverse energy. Furthermore, the vaccine may have led to expansion of new anti-MUC1 CTLs that had been removed from the repertoire by clonal deletion.

Immunization with the DC/tumor cell fusions was shown to induce an immune response that is sufficient to achieve rejection of established metastases. Interestingly, induction of an antiMUC1 response that confers antitumor immunity was shown to have very minimal effect on normal secretory epithelia that express MUC1 at apical borders along ducts. A potential explanation for the selectivity of anti-MUC1 response against tumors could be low levels of MUC1 peptide major histocompatibility complex class I complexes on normal cells.

Induction of anti-MUC1 immunity with the FC vaccine is a highly effective approach for the treatment of MUC1-positive MM cells in xenograft models.[72]. Indeed, in phase I clinical studies of patients with hematological malignancies such as AML and MM, and solid tumors, vaccination with DC/tumor fusions was well tolerated, induced the expansion of tumor specific $\mathrm{T}$ cells, and resulted in disease regression or stabilization in patients with advanced disease.[73-76]. The DC/tumor fusion vaccine has demonstrated striking efficacy in AML. In an immunocompetent murine model, vaccination with DC/AML fusions eradicates leukemic engraftment without evidence of autoimmunity. We are completing a clinical trial in which patients that achieve first or second remission following standard chemotherapy undergo serial vaccination with DC/AML fusions. Remarkably, despite a median age of 60 and high or intermediate risk factors present in all but one patient, $75 \%$ of the initial cohort of 16 patients remains free of disease with a median follow up of four years.[77].

Performing similar studies in CTCL and other hematological malignancies that highly express MUC1 is warranted with the goal of preventing recurrence in the setting of minimal residual disease.

\section{Conclusions}

Although widely recognized as an epithelial membrane protein that is upregulated in carcinomas, we and other groups have found MUC1 and specifically MUC1-C to be aberrantly overexpressed in various hematologic malignancies, including lymphomas, leukemias, and MM. In hematological malignancies, MUC1-C is associated with various pathways linked to disease pathogenesis, such as Wnt/ק-catenin, FLT3, BCR/ABL, NFkB, and others.[24]. Aberrant expression of MUC1-C is sufficient to induce transformation and block cellular death in response to genotoxic, oxidative, and hypoxic stress.

The interaction between the MUC1-C subunit and its multiple effectors is associated with malignant transformation. This finding suggests that MUC1-C is a potential target for cancer treatment. A cell penetrating peptide has been developed that binds to the MUC1-C CQC motif and blocks MUC1-C homodimerization and function. The inhibition of MUC1-C oligomerization in tumor cells results in decreased translocation to the cell nucleus, increase in intracellular reactive oxygen levels, activation of the DNA damage response, S phase arrest, loss of cell membrane integrity, and cellular necrosis/apoptosis.[17,30,52,53]. A 
phase I trial of the MUC1-C inhibitor, GO-203 has recently been completed in patients with refractory solid tumors to define the maximum tolerated dose. A phase I clinical trial is currently underway with the MUC1-C inhibitor, GO-203, in patients with relapsed/ refractory AML.

MUC1-C inhibition is also highly synergistic with other agents used to treat hematologic malignancies such as AraC, imatinib, bortezomib, and midostaurin/PKC412 in multiple in vitro and in vivo models. Further clinical and preclinical investigations of MUC1 inhibitors alone or in combination with other agents are warranted in the treatment of hematological malignancies.

\section{References}

1. Backstrom M, Link T, Olson FJ, et al. Recombinant MUC1 mucin with a breast cancer-like Oglycosylation produced in large amounts in Chinese-hamster ovary cells. Biochem J. 2003; 376:677-686. [PubMed: 12950230]

2. Brockhausen I, Yang JM, Burchell J, et al. Mechanisms underlying aberrant glycosylation of MUC1 mucin in breast cancer cells. Eur J Biochem. 1995; 233:607-617. [PubMed: 7588808]

3. Li Y, Liu D, Chen D, et al. Human DF3/MUC1 carcinoma-associated protein functions as an oncogene. Oncogene. 2003; 22:6107-6110. [PubMed: 12955090]

4. Raina D, Agarwal P, Lee J, et al. Characterization of the MUC1-C Cytoplasmic Domain as a Cancer Target. PLoS One. 2015; 10:e0135156. [PubMed: 26267657]

5. Raina D, Kosugi M, Ahmad R, et al. Dependence on the MUC1-C oncoprotein in non-small cell lung cancer cells. Mol Cancer Ther. 2011; 10:806-816. [PubMed: 21421804]

6. Rajabi H, Ahmad R, Jin C, et al. MUC1-C oncoprotein confers androgen-independent growth of human prostate cancer cells. Prostate. 2012; 72:1659-1668. [PubMed: 22473899]

7. Ye Q, Yan Z, Liao X, et al. MUC1 induces metastasis in esophageal squamous cell carcinoma by upregulating matrix metalloproteinase 13. Lab Invest. 2011; 91:778-787. [PubMed: 21339746]

8. Kufe DW. Mucins in cancer: function, prognosis and therapy. Nat Rev Cancer. 2009; 9:874-885. [PubMed: 19935676]

9. Kufe DW. MUC1-C oncoprotein as a target in breast cancer: activation of signaling pathways and therapeutic approaches. Oncogene. 2013; 32:1073-1081. [PubMed: 22580612]

10. Jain S, Stroopinsky D, Yin L, et al. Mucin 1 is a potential therapeutic target in cutaneous T-cell lymphoma. Blood. 2015; 126:354-362. [PubMed: 26048911]

11. Brossart P, Schneider A, Dill P, et al. The epithelial tumor antigen MUC1 is expressed in hematological malignancies and is recognized by MUC1-specific cytotoxic T-lymphocytes. Cancer Res. 2001; 61:6846-6850. [PubMed: 11559560]

12. Cloosen S, Gratama J, van Leeuwen EB, et al. Cancer specific Mucin-1 glycoforms are expressed on multiple myeloma. Br J Haematol. 2006; 135:513-516. [PubMed: 17010104]

13. Hasegawa H, Komoda M, Yamada Y, et al. Aberrant overexpression of membrane-associated mucin contributes to tumor progression in adult T-cell leukemia/lymphoma cells. Leuk Lymphoma. 2011; 52:1108-1117. [PubMed: 21599593]

14. Kawano T, Ahmad R, Nogi H, et al. MUC1 oncoprotein promotes growth and survival of human multiple myeloma cells. Int J Oncol. 2008; 33:153-159. [PubMed: 18575761]

15. Schlom J. The MUC1-C oncoprotein as a target in hematologic malignancies. Cancer Biol Ther. 2010; 10:492-494. [PubMed: 20716962]

16. Stroopinsky D, Rosenblatt J, Ito K, et al. MUC1 is a potential target for the treatment of acute myeloid leukemia stem cells. Cancer Res. 2013; 73:5569-5579. [PubMed: 23867470]

17. Yin L, Ahmad R, Kosugi M, et al. Survival of human multiple myeloma cells is dependent on MUC1 C-terminal transmembrane subunit oncoprotein function. Mol Pharmacol. 2010; 78:166174. [PubMed: 20444960] 
18. Yin L, Kufe D. MUC1-C oncoprotein blocks terminal differentiation of chronic myelogenous leukemia cells by a ROS-mediated mechanism. Genes Cancer. 2011; 2:56-64. [PubMed: 21643558]

19. Fatrai S, Schepers H, Tadema H, et al. Mucin1 expression is enriched in the human stem cell fraction of cord blood and is upregulated in majority of the AML cases. Exp Hematol. 2008; 36:1254-1265. [PubMed: 18640764]

20. Kufe DW. Functional targeting of the MUC1 oncogene in human cancers. Cancer Biol Ther. 2009; 8:1197-1203. [PubMed: 19556858]

21. Liu S, Yin L, Stroopinsky D, et al. MUC1-C oncoprotein promotes FLT3 receptor activation in acute myeloid leukemia cells. Blood. 2014; 123:734-742. [PubMed: 24282218]

22. Schroeder JA, Adriance MC, Thompson MC, et al. MUC1 alters beta-catenin-dependent tumor formation and promotes cellular invasion. Oncogene. 2003; 22:1324-1332. [PubMed: 12618757]

23. Huang L, Chen D, Liu D, et al. MUC1 oncoprotein blocks glycogen synthase kinase 3betamediated phosphorylation and degradation of beta-catenin. Cancer Res. 2005; 65:10413-10422. [PubMed: 16288032]

24. Ahmad R, Raina D, Joshi MD, et al. MUC1-C oncoprotein functions as a direct activator of the nuclear factor-kappaB p65 transcription factor. Cancer Res. 2009; 69:7013-7021. [PubMed: 19706766]

25. Ren J, Agata N, Chen D, et al. Human MUC1 carcinoma-associated protein confers resistance to genotoxic anticancer agents. Cancer Cell. 2004; 5:163-175. [PubMed: 14998492]

26. Ren J, Bharti A, Raina D, et al. MUC1 oncoprotein is targeted to mitochondria by heregulininduced activation of c-Src and the molecular chaperone HSP90. Oncogene. 2006; 25:20-31. [PubMed: 16158055]

27. Ahmad R, Alam M, Rajabi H, et al. The MUC1-C oncoprotein binds to the BH3 domain of the pro-apoptotic BAX protein and blocks BAX function. J Biol Chem. 2012; 287:20866-20875. [PubMed: 22544745]

28. Alam M, Rajabi H, Ahmad R, et al. Targeting the MUC1-C oncoprotein inhibits self-renewal capacity of breast cancer cells. Oncotarget. 2014; 5:2622-2634. [PubMed: 24770886]

29. Sahraei M, Roy LD, Curry JM, et al. MUC1 regulates PDGFA expression during pancreatic cancer progression. Oncogene. 2012; 31:4935-4945. [PubMed: 22266848]

30. Nath S, Daneshvar K, Roy LD, et al. MUC1 induces drug resistance in pancreatic cancer cells via upregulation of multidrug resistance genes. Oncogenesis. 2013; 2:e51. [PubMed: 23774063]

31. Raina D, Uchida Y, Kharbanda A, et al. Targeting the MUC1-C oncoprotein downregulates HER2 activation and abrogates trastuzumab resistance in breast cancer cells. Oncogene. 2014; 33:34223431. [PubMed: 23912457]

32. Michal Bar-Natan KL, Coll MD, Stroopinsky D, et al.Bone marrow stroma protects myeloma cells from cytotoxic damage via induction of the oncoprotein MUC1. Blood (Suppl). 2014; 124 Abstract 3378.

33. Dyomin VG, Palanisamy N, Lloyd KO, et al. MUC1 is activated in a B-cell lymphoma by the $\mathrm{t}(1 ; 14)(\mathrm{q} 21 ; \mathrm{q} 32)$ translocation and is rearranged and amplified in B-cell lymphoma subsets. Blood. 2000; 95:2666-2671. [PubMed: 10753849]

34. Yin L, Wu Z, Avigan D, et al. MUC1-C oncoprotein suppresses reactive oxygen species-induced terminal differentiation of acute myelogenous leukemia cells. Blood. 2011; 117:4863-4870. [PubMed: 21422470]

35. Kawano T, Ito M, Raina D, et al. MUC1 oncoprotein regulates Bcr-Abl stability and pathogenesis in chronic myelogenous leukemia cells. Cancer Res. 2007; 67:11576-11584. [PubMed: 18089786]

36. Yin L, Ahmad R, Kosugi M, et al. Terminal differentiation of chronic myelogenous leukemia cells is induced by targeting of the MUC1-C oncoprotein. Cancer Biol Ther. 2010; 10:483-491. [PubMed: 20592495]

37. Yin L, Li Y, Ren J, et al. Human MUC1 carcinoma antigen regulates intracellular oxidant levels and the apoptotic response to oxidative stress. J Biol Chem. 2003; 278:35458-35464. [PubMed: 12826677] 
38. Yin L, Kosugi M, Kufe D. Inhibition of the MUC1-C oncoprotein induces multiple myeloma cell death by down-regulating TIGAR expression and depleting NADPH. Blood. 2012; 119:810-816. [PubMed: 22117045]

39. Zhou Y, Rajabi H, Kufe D. Mucin 1 C-terminal subunit oncoprotein is a target for small-molecule inhibitors. Mol Pharmacol. 2011; 79:886-893. [PubMed: 21346142]

40. Gorrini C, Harris IS, Mak TW. Modulation of oxidative stress as an anticancer strategy. Nat Rev Drug Discov. 2013; 12:931-947. [PubMed: 24287781]

41. Bensaad K, Tsuruta A, Selak MA, et al. TIGAR, a p53-inducible regulator of glycolysis and apoptosis. Cell. 2006; 126:107-120. [PubMed: 16839880]

42. Yin L, Kufe T, Avigan D, et al. Targeting MUC1-C is synergistic with bortezomib in downregulating TIGAR and inducing ROS-mediated myeloma cell death. Blood. 2014; 123:29973006. [PubMed: 24632713]

43. Luisi-DeLuca C, Mitchell T, Spriggs D, et al. Induction of terminal differentiation in human K562 erythroleukemia cells by arabinofuranosylcytosine. J Clin Invest. 1984; 74:821-827. [PubMed: 6590568]

44. Konopleva M, Tsao T, Ruvolo P, et al. Novel triterpenoid CDDO-Me is a potent inducer of apoptosis and differentiation in acute myelogenous leukemia. Blood. 2002; 99:326-335. [PubMed: 11756188]

45. Rajabi H, Ahmad R, Jin C, et al. MUC1-C oncoprotein induces TCF7L2 transcription factor activation and promotes cyclin D1 expression in human breast cancer cells. J Biol Chem. 2012; 287:10703-10713. [PubMed: 22318732]

46. Bouillez A, Rajabi H, Pitroda S, et al. Inhibition of MUC1-C suppresses MYC expression and attenuates malignant growth in KRAS mutant lung adenocarcinomas. Cancer Res. 2016; 76:15381548. [PubMed: 26833129]

47. Ysebaert L, Chicanne G, Demur C, et al. Expression of beta-catenin by acute myeloid leukemia cells predicts enhanced clonogenic capacities and poor prognosis. Leukemia. 2006; 20:1211-1216. [PubMed: 16688229]

48. Wang Y, Krivtsov AV, Sinha AU, et al. The Wnt/beta-catenin pathway is required for the development of leukemia stem cells in AML. Science. 2010; 327:1650-1653. [PubMed: 20339075]

49. Tagde A, Rajabi H, Bouillez A, et al. MUC1-C drives MYC in multiple myeloma. Blood. 2016; 127:2587-2597. [PubMed: 26907633]

50. Rosnet O, Buhring HJ, Marchetto S, et al. Human FLT3/FLK2 receptor tyrosine kinase is expressed at the surface of normal and malignant hematopoietic cells. Leukemia. 1996; 10:238248. [PubMed: 8637232]

51. Raina D, Ahmad R, Kumar S, et al. MUC1 oncoprotein blocks nuclear targeting of c-Abl in the apoptotic response to DNA damage. EMBO J. 2006; 25:3774-3783. [PubMed: 16888623]

52. Li Y, Kuwahara H, Ren J, et al. The c-Src tyrosine kinase regulates signaling of the human DF3/ MUC1 carcinoma-associated antigen with GSK3 beta and beta-catenin. J Biol Chem. 2001; 276:6061-6064. [PubMed: 11152665]

53. Li Y, Bharti A, Chen D, et al. Interaction of glycogen synthase kinase 3beta with the DF3/MUC1 carcinoma-associated antigen and beta-catenin. Mol Cell Biol. 1998; 18:7216-7224. [PubMed: 9819408]

54. Ramasamy S, Duraisamy S, Barbashov S, et al. The MUC1 and galectin-3 oncoproteins function in a microRNA-dependent regulatory loop. Mol Cell. 2007; 27:992-1004. [PubMed: 17889671]

55. Li Y, Ren J, Yu W, et al. The epidermal growth factor receptor regulates interaction of the human DF3/MUC1 carcinoma antigen with c-Src and beta-catenin. J Biol Chem. 2001; 276:3523935242. [PubMed: 11483589]

56. Li Y, Yu WH, Ren J, et al. Heregulin targets gamma-catenin to the nucleolus by a mechanism dependent on the DF3/MUC1 oncoprotein. Mol Cancer Res. 2003; 1:765-775. [PubMed: 12939402]

57. Wei X, Xu H, Kufe D. Human MUC1 oncoprotein regulates p53-responsive gene transcription in the genotoxic stress response. Cancer Cell. 2005; 7:167-178. [PubMed: 15710329] 
58. Wei X, Xu H, Kufe D. MUC1 oncoprotein stabilizes and activates estrogen receptor alpha. Mol Cell. 2006; 21:295-305. [PubMed: 16427018]

59. Druker BJ. Translation of the Philadelphia chromosome into therapy for CML. Blood. 2008; 112:4808-4817. [PubMed: 19064740]

60. Lowenberg B. Minimal residual disease in chronic myeloid leukemia. N Engl J Med. 2003; 349:1399-1401. [PubMed: 14534331]

61. Ren R. Mechanisms of BCR-ABL in the pathogenesis of chronic myelogenous leukaemia. Nat Rev Cancer. 2005; 5:172-183. [PubMed: 15719031]

62. Palumbo A, Anderson K. Multiple myeloma. N Engl J Med. 2011; 364:1046-1060. [PubMed: 21410373]

63. Kuhn DJ, Berkova Z, Jones RJ, et al. Targeting the insulin-like growth factor-1 receptor to overcome bortezomib resistance in preclinical models of multiple myeloma. Blood. 2012; 120:3260-3270. [PubMed: 22932796]

64. Myrna Rita Nahas DS, Rajabi H, Tagde A, et al. MUC1 inhibition overcomes chemotherapy resistance in acute myeloid leukemia. Blood (Suppl). 2015; 126 Abstract 2473.

65. Brossart P, Heinrich KS, Stuhler G, et al. Identification of HLA-A2-restricted T-cell epitopes derived from the MUC1 tumor antigen for broadly applicable vaccine therapies. Blood. 1999; 93:4309-4317. [PubMed: 10361129]

66. Pol J, Bloy N, Buque A, et al. Trial Watch: Peptide-based anticancer vaccines. Oncoimmunology. 2015; 4:e974411. [PubMed: 26137405]

67. Tarp MA, Sorensen AL, Mandel U, et al. Identification of a novel cancer-specific immunodominant glycopeptide epitope in the MUC1 tandem repeat. Glycobiology. 2007; 17:197209. [PubMed: 17050588]

68. Rivalland G, Loveland B, Mitchell P. Update on Mucin-1 immunotherapy in cancer: a clinical perspective. Expert Opin Biol Ther. 2015; 15:1773-1787. [PubMed: 26453294]

69. Arlen PM, Gulley JL, Madan RA, et al. Preclinical and clinical studies of recombinant poxvirus vaccines for carcinoma therapy. Crit Rev Immunol. 2007; 27:451-462. [PubMed: 18197807]

70. Carmon L, Avivi I, Kovjazin R, et al. Phase I/II study exploring ImMucin, a pan-major histocompatibility complex, anti-MUC1 signal peptide vaccine, in multiple myeloma patients. Br J Haematol. 2015; 169:44-56. [PubMed: 25496030]

71. Gong J, Chen D, Kashiwaba M, et al. Reversal of tolerance to human MUC1 antigen in MUC1 transgenic mice immunized with fusions of dendritic and carcinoma cells. Proc Natl Acad Sci USA. 1998; 95:6279-6283. [PubMed: 9600956]

72. Gong J, Koido S, Chen D, et al. Immunization against murine multiple myeloma with fusions of dendritic and plasmacytoma cells is potentiated by interleukin 12. Blood. 2002; 99:2512-2517. [PubMed: 11895787]

73. Avigan D. Dendritic cell-tumor fusion vaccines for renal cell carcinoma. Clin Cancer Res. 2004; 10:6347S-6352S. [PubMed: 15448029]

74. Avigan DE, Vasir B, George DJ, et al. Phase I/II study of vaccination with electrofused allogeneic dendritic cells/autologous tumor-derived cells in patients with stage IV renal cell carcinoma. J Immunother. 2007; 30:749-761. [PubMed: 17893567]

75. Rosenblatt J, Avivi I, Vasir B, et al. Vaccination with dendritic cell/tumor fusions following autologous stem cell transplant induces immunologic and clinical responses in multiple myeloma patients. Clin Cancer Res. 2013; 19:3640-3648. [PubMed: 23685836]

76. Rosenblatt J, Vasir B, Uhl L, et al. Vaccination with dendritic cell/tumor fusion cells results in cellular and humoral antitumor immune responses in patients with multiple myeloma. Blood. 2011; 117:393-402. [PubMed: 21030562]

77. Jacalyn Rosenblatt RMS, Uhl L, Neuberg D, et al. DC/Aml fusion cell vaccination administered to AML patients who achieve a complete remission potently expands leukemia reactive $\mathrm{T}$ cells and is associated with durable remissions. Blood (Suppl). 2015; 126 Abstract 2549. 


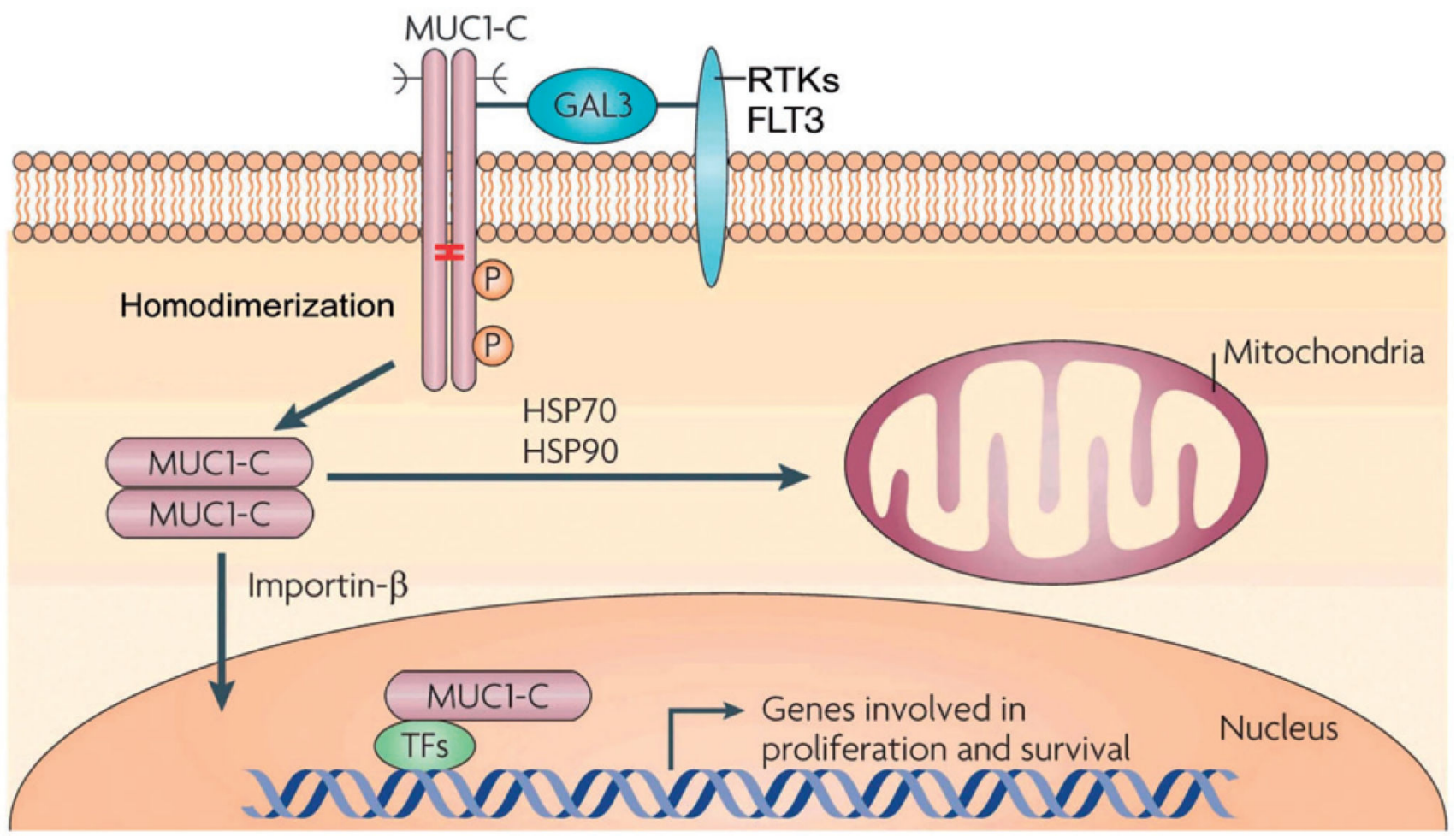

Figure 1.

Activation of the MUC1-C subunit in hematopoietic malignancies. MUC1-C homodimers form complexes with FLT3 and other RTKs at the cell membrane that are mediated at least in part by galectin-3 (GAL3). Internalization of MUC1-C is associated with its import into the nucleus by an importin- $\beta$-mediated mechanism. The association of MUC1-C with certain transcription factors (TFs), such as NF- $\kappa B$ p65 and $\beta$-catenin/TCF4, promotes the expression of genes involved in self-renewal and survival. MUC1-C is also transported to mitochondria in a complex with HSP70 and HSP90, where it blocks cell death. GO-203 blocks MUC1-C homodimerization, nuclear import, and oncogenic function. (Modified from Figure 5 in ref. [8]). 


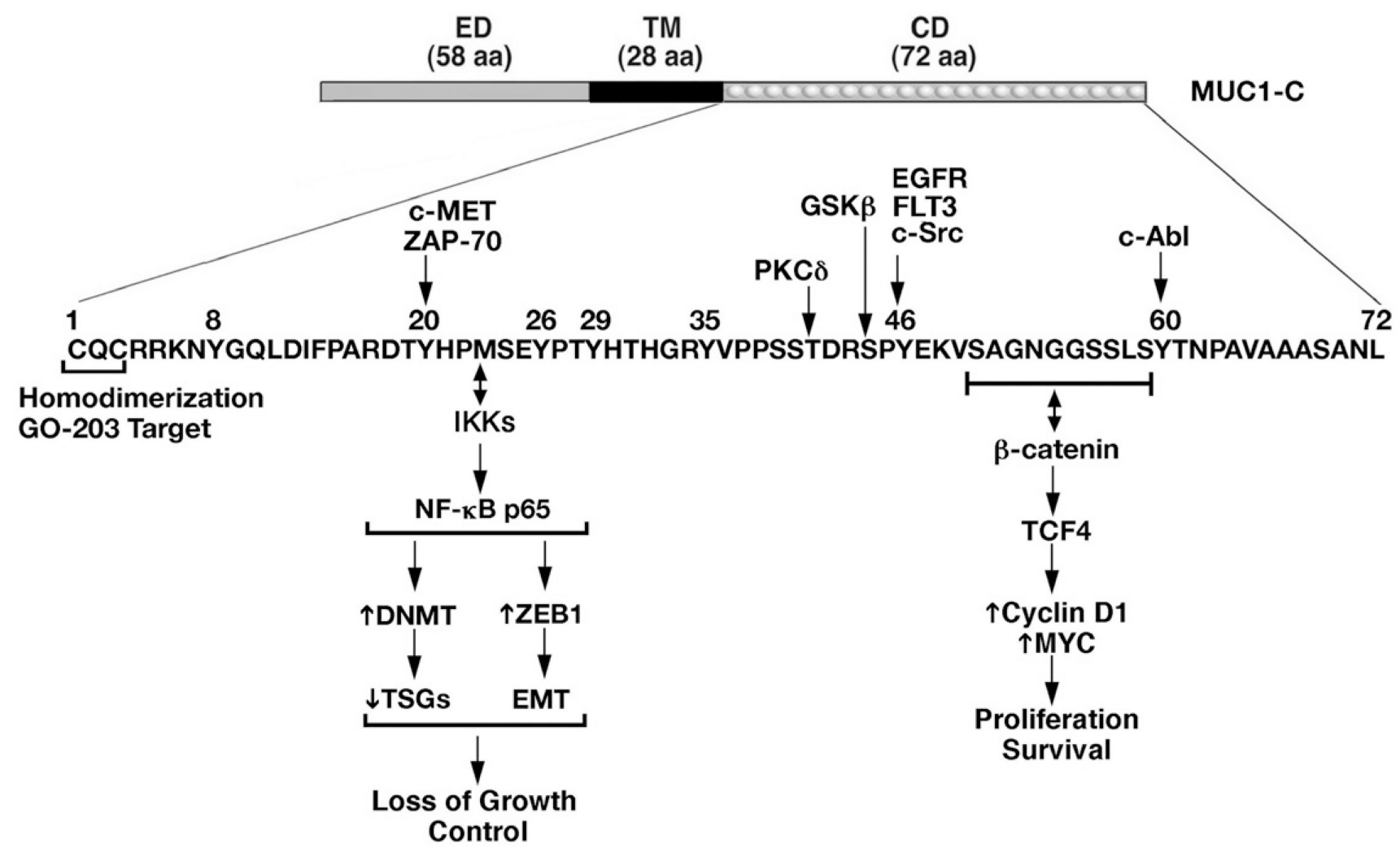

Figure 2.

Schematic representation of the MUC1-C subunit. MUC1-C consists of a 58 aa non-shed extracellular domain (ED), a 28 aa transmembrane domain (TM) and a 72 aa cytoplasmic domain (CD). The MUC1-C cytoplasmic domain contains a CQC motif that is necessary for MUC1-C homodimerization, localization to the nucleus and oncogenic function. The MUC1-C CQC motif is the target of the GO-203 inhibitor. Highlighted are selected phosphorylation sites and binding regions for activation of the IKK $\rightarrow$ NF-kB p65 and the WNT/b-catenin/TCF4 pathways that promote the malignant phenotype. DNMT: DNA methyltransferase; TSG: tumor suppressor gene. 